\title{
PROBABILISTIC CONVERGENCE SPACES AND GENERALIZED METRIC SPACES
}

\author{
PAUL BROCK \\ Washington State University \\ Pullman, WA. 99164-3113 \\ (Received June 25, 1996)
}

\begin{abstract}
The category $P P R S(\Delta)$, whose objects are probabilistic pretopological spaces which satisfy an axiom $(\Delta)$ and whose morphisms are continuous mappings, is introduced. Categories consisting of generalized metric spaces as objects and contraction mappings as morphisms are embedded as full subcategories of $\operatorname{PPRS}(\Delta)$. The embeddings yield a description of metric spaces and their most natural generalizations entirely in terms of convergence criteria.
\end{abstract}

KEY WORDS AND PHRASES. Generalized metric space, probabilistic convergence space, $t$-norm, diagonal axiom, functor, isomorphism

1991 MATHEMATICS SUBJECT CLASSIFICATION CODE.

54 B 30, 54 A 05, 54 A 20, 54 E 70

\section{Introduction}

The category pqs-MET ${ }^{\infty}$, with extended pseudo-quasi-semi-metric spaces as objects and contraction mappings as morphisms, is the most general metric category we shall consider. An object $(X, d)$ in pqs-MET $T^{\infty}$ consists of a set $X$ and a distance function $d: X \times X \rightarrow[0, \infty]$ which satisfies the single axiom $d(x, x)=0$, for all $x \in X$. By allowing $d$ to assume the value $\infty$, we obtain a well-behaved category; i.e., the category pqs-MET ${ }^{\infty}$ is topological.

In this paper, we study $p q s-M E T^{\infty}$ as a full subcategory of $P P R S$, the category of probabilistic pretopological spaces. The latter spaces were first introduced by G. Richardson and D. Kent [14] as generalizations of probabilistic metric spaces (see [15], [16]). The existence of an isomorphism between pqs-MET ${ }^{\infty}$ and a subcategory of PPRS follows from results established in [3] and [10]. Among other important full subcategories of PPRS are the categories PRTOP of pretopological spaces and TOP of topological spaces, each of which is embedded in PPRS in an obvious way.

Our goal is to find simple axioms for objects in PPRS, based entirely on convergence criteria, which characterize pqs-MET ${ }^{\infty}$ (considered as a subcategory of $P P R S$ ) along with its most important full subcategories: $p q-M E T^{\infty}, p-M E T^{\infty}$, and $M E T^{\infty}$.

It follows from the results of [2] that there is a family of isomorphic embeddings of pqs-MET into PPRS which depend on the choice of an order reversing homeomorphism $S$. However the pgs-MET ${ }^{\infty}$ objects in PPRS are characterized by means of an axiom $(\Delta)$ which is independent the $t$-norm derived from $S$. On the other hand, the axioms $F_{T}$ and $R_{T}$ which, along with $(\Delta)$, characterize the subcategories $p q-M E T^{\infty}$ and $p-M E T^{\infty}$ infinity in $P P R S$ do depend on $T$. 
It is interesting to observe that the notion of "t-norm," whose original purpose was to define the "triangle inequality" for probabilistic metric spaces, also arises naturally when characterizing those "metric objects" in PPRS which depend on the triangle inequality, notably the pseudo-quasi metric objects.

\section{Convergence Spaces}

Let $X$ be a set, $\mathrm{F}(X)$ the set of all (proper) filters on $X, \mathrm{U}(X)$ the set of all ultrafilters filters on $X$, and $2^{X}$ the set of all subsets of $X$. For $x \in X$, denote by $\dot{x}$ the fixed ultrafilter generated by $\{x\}$.

Definition 2.1 A convergence structure $q$ on a set $X$ is a function $q: F(X) \rightarrow 2^{X}$ satisfying:

$\left(C_{1}\right) x \in q(\dot{x})$, for all $x \in X$;

$\left(C_{2}\right) \mathcal{F} \leq \mathcal{G} \Rightarrow q(\mathcal{F}) \subseteq q(\mathcal{G})$

$\left(C_{3}\right) x \in q(\mathcal{F}) \Rightarrow x \in q(\mathcal{F} \cap \dot{x})$.

We interpret $x \in q(\mathcal{F})$ to mean " $\mathcal{F} q$-converges to $x . "$ The idea of "convergence" may be more intuitively conveyed by writing " $\mathcal{F} \stackrel{q}{\rightarrow} x$." If $q$ is a convergence structure on $X$, then the pair $(X, q)$ is called a convergence space. A function $f:(X, q) \rightarrow(Y, p)$ between convergence spaces is continuous if $f(\mathcal{F}) \stackrel{p}{\rightarrow} f(x)$ whenever $\mathcal{F} \stackrel{q}{\rightarrow} x$. If $p$ and $q$ are convergence structures on $X$ and $f:(X, q) \rightarrow(X, p)$ is continuous, where $f$ is the identity map on $X$, then we write $p \leq q$ ( $p$ is coarser than $q$, or $q$ is finer than $p$ ).

There is associated with each convergence space $(X, q)$, a closure operator $c l_{q}$ and an interior operator $I_{q}$. These are defined for each $A \in 2^{X}$ by the following:

$\operatorname{cl}_{q} A=\{x \in X: \exists \mathcal{F} \stackrel{q}{\rightarrow} x$ such that $A \in \mathcal{F}\}$;

$I_{q} A=\{x \in A: \mathcal{F} \stackrel{q}{\rightarrow} x \Rightarrow A \in \mathcal{F}\}$.

The $q$-closure and $q$-interior can also be described using only ultrafilters in the above definitions.

If $\mathcal{F}$ is a filter on $X, c l_{q} \mathcal{F}$ denotes the filter generated by $\left\{c l_{q} F: F \in \mathcal{F}\right\}$. A convergence space $(X, q)$ is defined to be regular if $\mathcal{F} \stackrel{q}{\rightarrow} x$ implies $c l_{q} \mathcal{F} \stackrel{q}{\rightarrow} x$.

For every $x \in X$, let

$$
\mathcal{V}_{q}(x)=\left\{V \subseteq X: x \in I_{q} V\right\}
$$

$\mathcal{V}_{q}(x)$ is called the $q$-neighborhood filter at $x$. The $q$-neighborhood filter can also be described as the intersection of all ultrafilters which $q$-converge to $x$.

We have three additional convergence axioms to consider:

$\left(C_{\mathbf{4}}\right) q(\mathcal{F} \cap \mathcal{G})=q(\mathcal{F}) \cap q(\mathcal{G})$, for all $\mathcal{F}, \mathcal{G} \in \mathbf{F}(X)$;

$\left(C_{5}\right)$ For each $\mathcal{F} \in \mathbf{F}(X), x \in q(\mathcal{F})$ iff $x \in q(\mathcal{G})$, for every ultrafilter $\mathcal{G} \geq \mathcal{F}$;

$\left(C_{6}\right) x \in q\left(\mathcal{V}_{q}(x)\right)$, for all $x \in X$.

A convergence structure $q$ which satisfies $\left(C_{4}\right)$ (respectively, $\left(C_{5}\right),\left(C_{6}\right)$ ) is called a limit structure (respectively, pseudo-topology, pretopology). Observe that pretopology $\Rightarrow$ pseudo-topology $\Rightarrow$ limit 
structure $\Rightarrow$ convergence structure. A set $A \subseteq X$ is $q$-open if $I_{q} A=A$. A pretopology $q$ is a topology if every neighborhood filter $\mathcal{V}_{q}(x)$ has a filter base consisting of sets which are q-open.

It is interesting that the convergence properties "regular" and "topological" are in a very natural sense dual to each other, since they can be characterized by means of dual axioms, which we call $F$ and $R$, dive to C.H. Cook and H.R. Fischer [3]. Let $X$ and $J$ be non-empty sets, $\mathcal{F} \in F(J)$, and $\sigma: J \rightarrow \mathbf{F}(X)$. We define

$$
\kappa \sigma \mathcal{F}=\bigcup_{F \in \mathcal{F}} \bigcap_{y \in F} \sigma(y)
$$

$\kappa$ is called the "compression operator for $\mathcal{F}$ relative to $\sigma$." Note that if $\mathcal{F} \in \mathrm{U}(J)$, and $\sigma(y) \in \mathrm{U}(X)$ for all $y \in J$, then $\kappa \sigma \mathcal{F} \in \mathrm{U}(X)$. We can now define the axioms $F$ and $R$.

$F$ : Let $J$ be a non-empty set, $\psi: J \rightarrow X$, and let $\sigma: J \rightarrow \mathbf{F}(X)$ have the property that $\sigma(y) \stackrel{q}{\rightarrow} \psi(y)$, for all $y \in J$. If $\mathcal{F} \in \mathbf{F}(J)$ is such that $\psi(\mathcal{F}) \stackrel{q}{\rightarrow} x$, then $\kappa \sigma \mathcal{F} \stackrel{\mathfrak{q}}{\rightarrow} x$.

$R$ : Let $J$ be a non-empty set, $\psi: J \rightarrow X$, and let $\sigma: J \rightarrow F(X)$ have the property that $\sigma(y) \stackrel{q}{\rightarrow} \psi(y)$, for all $y \in J$. If $\mathcal{F} \in \mathbf{F}(J)$ is such that $\kappa \sigma \mathcal{F} \stackrel{q}{\rightarrow} x$, then $\psi(\mathcal{F}) \stackrel{q}{\rightarrow} x$.

The next proposition summarizes previously mentioned results pertaining to these axioms. The first assertion is proved in [14], the second in [1] and [5].

Proposition 2.2 Let $(X, q)$ be a convergence space.

(1) $(X, q)$ is topological if and only if it satisfies $F$.

(2) $(X, q)$ is regular if and only if it satisfies $R$.

Let $\tilde{F}$ and $\tilde{R}$ denote the axioms obtained when ${ }^{"} \mathbf{F}(X)^{n}$ and ${ }^{~} \mathbf{F}(J)^{n}$ are replaced by ${ }^{~} \mathrm{U}(X)$ " and ${ }^{\text {" }} \mathrm{U}(J)^{n}$ in $F$ and $R$, respectively. Obviously, $F \Rightarrow \tilde{F}$ and $R \Rightarrow \tilde{R}$. Part (1) of the next proposition is proved in [17]. Part (2) is proved in [4]

Proposition 2.3 Let $(X, q)$ be a pseudotopological convergence space.

(1) $(X, q)$ is topological if and only if it satisfies $\tilde{F}$.

(2) $(X, q)$ is regular if and only if it satisfies $\tilde{R}$.

\section{The ( $\beta$ ) Axiom for Convergence Spaces.}

In this section, we introduce the convergence space axiom $(\beta)$, and give equivalent characterizations of $\tilde{F}$ and $\tilde{R}$ for convergence spaces $(X, q)$ which satisfy $(\beta)$. It should be mentioned that $(\beta)$ is a very strong axiom; the only $\mathbf{T}_{1}$ space which satisfies $(\beta)$ is the discrete topology. Some additional terminology will be useful.

Definitions 3.1 Let $(X, q)$ be a convergence space, and let $x, y, z \in X .(X, q)$ is said to be symmetric, if $\dot{y} \stackrel{q}{\rightarrow} x$ whenever $\dot{x} \stackrel{q}{\rightarrow} y$;

transitive, if $\dot{x} \stackrel{q}{\rightarrow} z$ whenever $\dot{x} \stackrel{q}{\rightarrow} y$ and $\dot{y} \stackrel{q}{\rightarrow} z$;

skew transitive, if $\dot{x} \stackrel{q}{\rightarrow} z$ whenever $\dot{y} \stackrel{q}{\rightarrow} x$ and $\dot{y} \stackrel{q}{\rightarrow} z$. 
Our first proposition provides an equivalence for skew transitivity. The proof is easy.

Proposition 3.2 A convergence space $(X, q)$ is skew transitive iff it is both transitive and symmetric.

$(\beta):$ For all $\mathcal{U} \in \mathrm{U}(X), \mathcal{U} \stackrel{q}{\rightarrow} x \Longleftrightarrow$ for all $U \in \mathcal{U}$, there exists $y \in U$ such that $\dot{y} \stackrel{q}{\rightarrow} x$.

In the next section, we will extend both $(\beta)$ and the following results to the setting of probabilistic convergence spaces.

Proposition 3.3 Let $(X, q)$ be a convergence space which satisfies $(\beta)$. Then $(X, q)$ satisfies $\tilde{F}$ iff $(X, q)$ is transitive.

Proof: Assume $\tilde{F}$ holds, let $J=X$, and $\psi=\operatorname{id} x$. Let $x, y, z \in X$, such that $\dot{x} \stackrel{q}{\rightarrow} y$ and $\dot{y} \stackrel{q}{\rightarrow} z$. Define $\sigma: X \rightarrow \mathrm{U}(X)$ as follows : $\sigma(w)=\dot{w}$ for all $w \neq y$, and $\sigma(y)=\dot{x}$. Thus, $\kappa \sigma(\dot{y})=\dot{x} \stackrel{q}{\rightarrow} z$.

To show the converse, let $J, \sigma, \psi$, and $\mathcal{F}$ be as in the statement of $\tilde{F}, \sigma(w) \stackrel{q}{\rightarrow} \psi(w)$ for all $w \in J$, and $\psi \mathcal{F} \stackrel{q}{\rightarrow} z$ for some $z \in X$. Let $D \in \kappa \sigma \mathcal{F}$. Recall that a filter base for $\kappa \sigma \mathcal{F}$ is given by

$$
\left\{\bigcup_{y \in F} U_{y}: F \in \mathcal{F} \text { and } U_{y} \in \sigma(y)\right\}
$$

Thus, $\bigcup_{y \in F} U_{y} \subseteq D$ for some $F \in \mathcal{F}$. By axiom $(\beta)$, there exists a $b \in F$ such that $\dot{\psi(b)} \stackrel{q}{\rightarrow} z$. Since $\sigma(b) \stackrel{\mathfrak{q}}{\rightarrow} \psi(b),(\beta)$ further implies that there exists a $x \in U_{b} \subseteq D$ such that $\dot{x} \stackrel{q}{\rightarrow} \psi(b)$. Hence, $\dot{x} \stackrel{q}{\rightarrow} z$ by transitivity. Since $D$ was chosen arbitrarily, $(\beta)$ implies $\kappa \sigma \mathcal{F} \stackrel{q}{\rightarrow} z$.

Proposition 3.4 Let $(X, q)$ be a convergence space which satisfies $(\beta)$. Then $(X, q)$ satisfies $\tilde{R}$ iff $(X, q)$ is skew transitive.

Proof: Assume $\tilde{R}$ holds, let $J=X$, and $\psi=\mathrm{id} x$. Let $x, y, z \in X$, such that $\dot{y} \stackrel{q}{\rightarrow} x$ and $\dot{y} \stackrel{q}{\rightarrow} z$. Define $\sigma: X \rightarrow U(X)$ as follows : $\sigma(w)=\dot{w}$ for all $w \neq x$ and $\sigma(x)=\dot{y}$; hence, $\kappa \sigma(\dot{x})=\dot{y} \stackrel{q}{\rightarrow} z$. Therefore, $\psi \dot{x}=\dot{x} \stackrel{q}{\rightarrow} z$.

For the converse, let $J, \psi, \sigma$, and $\mathcal{F}$ be as in $\tilde{R}, \sigma(w) \stackrel{q}{\rightarrow} \psi(w)$ for all $\dot{w} \in J$, and $\kappa \sigma \mathcal{F} \stackrel{q}{\rightarrow} z$. Let $F \in \mathcal{F}$ and let $A_{F}=\{x \in X: \dot{x} \stackrel{q}{\rightarrow} \psi(j)$, for some $j \in F\}$. If $X \backslash A_{F} \in \kappa \sigma \mathcal{F}$, then there exists $G \in \mathcal{F}$ such that $\bigcup_{w \in G} U_{w} \subseteq X \backslash A_{F}$, where $U_{w} \in \sigma(w)$. Let $b \in F \cap G$. Since $\sigma(b) \stackrel{q}{\rightarrow} \psi(b)$, there exists a $x_{b} \in U_{b} \subseteq X \backslash A_{F}$ such that $\dot{x}_{b} \stackrel{q}{\rightarrow} \psi(b)$, by axiom $(\beta)$. But this implies $x_{b} \in A_{F}$, which is a contradiction; therefore, $A_{F} \in \kappa \sigma \mathcal{F}$. Hence, there exists a $y \in A_{F}$ such that $\dot{y} \stackrel{q}{\rightarrow} z$, by $(\beta)$; but $\dot{y} \stackrel{q}{\rightarrow} \psi(j)$ for some $j \in F$, by definition of $A_{F}$. It follows that $\psi(j) \stackrel{q}{\rightarrow} z$, by skew transitivity. Since $F$ was chosen arbitrarily, $\psi \mathcal{F} \stackrel{q}{\rightarrow} z$, by $(\beta)$.

Corollary 3.5 Let $(X, q)$ be a convergence space which satisfies $(\beta)$. Then $(X, q)$ satisfies $\tilde{R}$ iff $(X, q)$ is transitive and symmetric.

Proof: Use Proposition 3.2. and Proposition 3.4. 


\section{Probabilistic Convergence Spaces}

Probabilistic convergence spaces have evolved from the study of probabilistic metric spaces and their generalizations (see [6], [7], [12], [15], [16]). A filter-based theory for such spaces was introduced in [14].

Let $I$ denote the unit interval $[0,1]$ in $R$.

Definition 3.1 A probabilistic convergence structure $q$ on $X$ is a function

$$
\mathrm{q}: \mathbf{F}(X) \times I \rightarrow 2^{X}
$$

satisfying:

(PCS1) : For each $\mu \in I, \mathbf{q}(\mathcal{F}, \mu)=q_{\mu}(\mathcal{F})$, where $q_{\mu} \in \mathbf{C}(X)$;

(PCS2) : If $\mu=0, q_{\mu}$ is the indiscrete topology;

(PCS3) : If $\mu \leq \nu \in I$, then $q_{\mu} \leq q_{\nu}$;

(PCS4) : For each $\mu \in I, q_{\mu}=\sup \left\{q_{\nu}: \nu<\mu\right\}$.

The condition (PCS4) of Definition 3.1 is called left-continuity.

We will generally write $q=\left(q_{\mu}\right)$, where $\mu$ is assumed to range through $I$. If $q$ is a probabilistic convergence structure on $X$, then $(X, q)$ is called a probabilistic convergence space. Essentially, a probabilistic convergence space may be regarded as a family of convergence spaces $\left\{\left(X, q_{\mu}\right): \mu \in I\right\}$. If a filter $\mathcal{F} q_{\mu}$-converges to a point $\mathrm{x}$, we say that "the probability that $\mathcal{F} \mathbf{q}$-converges to $\mathrm{x}$ is at least $\mu$." So q gives a rule for determining the probability that any given filter on $X$ converges to any given point in $X$. The probability that a filter $\mathcal{F}$ q-converge to $x$ is defined to be $\lambda=\sup \{\mu \in I: \mathcal{F} \stackrel{q \mu}{\rightarrow} x\}$.

If $(X, \mathbf{q})$ and $(Y, \mathbf{p})$ are probabilistic convergence spaces and $f: X \rightarrow Y$ is a mapping, then $f:(X, q) \rightarrow(Y, p)$ is said to be continuous if $f:\left(X, q_{\mu}\right) \rightarrow\left(Y, p_{\mu}\right)$ is continuous for all $\mu \in I$. The category with probabilistic convergence spaces as objects and continuous functions as morphisms is denoted by $P C S$. If $(X, q) \in|P C S|$ and each $q_{\mu}$ is a limit structure (respectively, pseudotopology, pretopology), then $(X, \mathbf{q})$ is called a probabilistic limit space (respectively, probabilistic pseudotopological space, probabilistic pretopological space), and the corresponding full subcategory of $P C S$ is denoted by $P L S$ (respectively, PPSS, PPRS). Note that PPRS $\subseteq P P S S \subseteq P L S \subseteq P C S$. A probabilistic convergence space $(X, q)$ is defined to be $T_{1}$ (respectively, $\left.\mathbf{T}_{\mathbf{2}}\right)$ if the convergence space $\left(X, q_{1}\right)$ is $\mathbf{T}_{1}$ (respectively, $\mathbf{T}_{2}$ ).

We next define "t-norm," a notion which is fundamental in the study of probabilistic metric spaces and their generalizations. For further information about this topic, the reader is referred to $[16]$.

Definition 4.1 A t-norm is a binary operation $T: I^{2} \rightarrow I$ which is associative, commutative, increasing in each variable, and satisfies: $T(\mu, 1)=\mu$, for all $\mu \in I$.

A $t$-norm $T$ is said to be left-continuous if $T(x, y)=\sup \{T(u, v): 0<u<x, 0<v<y\}$, for all $x, y \in(0,1]$. 
Let $\mathcal{T}$ be the set of all $t$-norms. A partial order on $\mathcal{T}$ is defined as follows:

$$
T \leq T^{\prime} \text { iff } T(\mu, \nu) \leq T^{\prime}(\mu, \nu) \text { for all }(\mu, \nu) \in I^{2} .
$$

The smallest $t$-norm, $\hat{T}$ is defined by

$$
\hat{T}(\mu, \nu)= \begin{cases}\mu, & \text { if } \nu=1 \\ \nu, & \text { if } \mu=1 \\ 0, & \text { otherwise. }\end{cases}
$$

The largest $t$-norm is $\check{T}$, defined by

$$
\check{T}(\mu, \nu)=\min \{\mu, \nu\}
$$

for all $(\mu, \nu) \in I^{2}$.

Let $(X, \mathrm{q})$ be a probabilistic convergence space, and $T \in \mathcal{T}$. We define two axioms for $(X, \mathrm{q})$ relative to $T$ which are derived in an obvious way from the axioms $F$ and $R$ of Section 2.

$F_{T}:$ Let $\mu, \nu \in I$. Let $J$ be any non-empty set, $\psi: J \rightarrow X$ and $\sigma: J \rightarrow \mathbf{F}(X)$ be such that $\sigma(y) \stackrel{q_{\nu}}{\rightarrow} \psi(y)$, for each $y \in J$. If $\mathcal{F} \in \mathbf{F}(J)$ and $\psi \mathcal{F} \stackrel{q_{\mu}}{\rightarrow} x$, then $\kappa \sigma \mathcal{F} \stackrel{q \mathcal{T}(\mu, \nu)}{\longrightarrow} x$.

$R_{T^{\prime}}:$ Let $\mu, \nu \in I$. Let $J$ be any non-empty set, $\psi: J \rightarrow X$ and $\sigma: J \rightarrow F(X)$ be such that $\sigma(y) \stackrel{q_{\nu}}{\rightarrow} \psi(y)$, for each $y \in J$. If $\mathcal{F} \in \mathbf{F}(J)$ and $\kappa \sigma \mathcal{F} \stackrel{q_{\mu}}{\rightarrow} x$, then $\psi \mathcal{F} \stackrel{q_{T(\mu, \nu)}}{\longrightarrow} x$.

Definitions 4.2 Let $T$ be a $t$-norm. If a probabilistic convergence space $(X, q)$ satisfies $F_{T}$, it is called $T$-topological. If $(X, q)$ satisfies $R_{T}$, it is called $T$-regular.

The proof of the following proposition appears in [2].

Proposition 4.3 Let $T$ be a $t$-norm, and let $(X, q)$ be a probabilistic convergence space. Then $(X, q)$ is $T$-regular iff, for all $\mu, \nu \in I, \mathcal{F} \stackrel{q_{\nu}}{\rightarrow} x$ implies $c l_{q_{\mu}} \mathcal{F}^{q_{T}(\mu, \nu)} \rightarrow$.

Similarly, for any $t$-norm $T$, we may derive the axioms $\tilde{F}_{T}$ and $\tilde{R}_{T}$ from the axioms $\tilde{F}$ and $\tilde{R}$, respectively. The next proposition is proved in [4].

Proposition 4.4 Let $(X, q)$ be a probabilistic pseudotopological space.

(1) $(X, \mathbf{q})$ satisfies $\tilde{F}_{T}$ iff $(X, \mathbf{q})$ satisfies $F_{T}$.

(2) $(X, \mathbf{q})$ satisfies $\tilde{R}_{T}$ iff $(X, \mathbf{q})$ is $R_{T}$.

For a fixed $t$-norm $T$, the full subcategory of PCS whose objects are $T$-topological is denoted by $F_{T} P C S$. The categories $R_{T} P C S, \tilde{F}_{T} P C S$, and $\tilde{R}_{T} P C S$ are defined analogously.

We conclude this section with the following simple result.

Proposition 4.5 Let $T$ be a $t$-norm, and let $(X, \mathbf{q})$ be a $T$-regular probabilistic convergence space. Then $(X, \mathbf{q})$ is $\mathbf{T}_{\mathbf{1}}$ iff $(X, \mathbf{q})$ is $\mathbf{T}_{\mathbf{2}}$.

Proof: Assume $(X, q)$ is $T_{1}$. Then $\left(X, q_{1}\right)$ is $T_{1}$, and by Proposition 4.3, $\mathcal{F} \stackrel{q_{1}}{\rightarrow} x$ implies $c l_{q_{1}} \mathcal{F} \stackrel{q_{T(1,1)}}{\rightarrow} x$. But $T(1,1)=1$, by Definition 4.1; hence, $\left(X, q_{1}\right)$ is regular, and so $\left(X, q_{1}\right)$ is $\mathbf{T}_{2}$. Thus, $(X, \mathbf{q})$ is $\mathbf{T}_{\mathbf{2}}$.

The converse is clear. 


\section{The $(\Delta)$ Axiom for Probabilistic Convergence Spaces}

We now extend the results of Section 3 to the setting of probabilistic convergence spaces.

Definitions 5.1 Let $(X, q)$ be a probabilistic convergence space, let $T$ be a $t$-norm and let $x, y, z \in$ $X .(X, \mathbf{q})$ is said to be symmetric : if, for every $\mu \in I, \dot{x} \stackrel{q_{\mu}}{\rightarrow} y$ implies $\dot{y} \stackrel{q_{\mu}}{\rightarrow} x$; $T$-transitive : if $\dot{x} \stackrel{q_{T(\mu, \nu)}}{\longrightarrow} z$ whenever $\dot{x} \stackrel{q_{\nu}}{\longrightarrow} y$ and $\dot{y} \stackrel{q_{\mu}}{\rightarrow} z$; skew $T$-transitive : if $\dot{x} \stackrel{q_{T(\mu, \nu)}}{\longrightarrow} z$ whenever $\dot{y} \stackrel{q_{\nu}}{\longrightarrow} x$ and $\dot{y} \stackrel{q_{\mu}}{\longrightarrow} z$.

Proposition 5.2 A probabilistic convergence space $(X, q)$ is skew $T$-transitive iff it is both $T$ transitive and component-wise symmetric.

Proof: Let $\mu \in I$, and assume $(X, q)$ is skew $T$-transitive. Then $\dot{z} \stackrel{q_{1}}{\rightarrow} z$ and $\dot{z} \stackrel{q_{\mu}}{\rightarrow} x$ implies $\dot{x} \stackrel{q_{T(1, \mu)}}{\longrightarrow} z$, or more simply, $\dot{z} \stackrel{q_{\mu}}{\longrightarrow} x$ implies $\dot{x} \stackrel{q_{\mu}}{\rightarrow} z$. Hence, $(X, \mathbf{q})$ is symmetric, and transitivity now follows easily.

The converse is straightforward.

The following axiom, denoted $(\Delta)$, extends the $(\beta)$ axiom of Section 3 to the setting of probabilistic convergence spaces. The $(\Delta)$ axiom will hold in those probabilistic convergence spaces which have a natural correspondence with pseudo-quasi-semi metric spaces.

$(\Delta)$ : For all $\mathcal{U} \in \mathbf{U}(X)$ and $\nu \in I, \mathcal{U} \stackrel{q_{\nu}}{\rightarrow} x \Longleftrightarrow$ for all $U \in \mathcal{U}$, and all $\mu<\nu$, there exists a $y \in U$ such that $\dot{y} \stackrel{q \mu}{\rightarrow} x$.

Propositions 5.3 and 5.4 extend the results of Propositions 3.3 and 3.4, repectively.

Proposition 5.3 Let $(X, q)$ be a probabilistic convergence space which satisfies $(\Delta)$. Then, for any left-continuous $t$-norm $T,(X, \mathbf{q})$ satisfies $\tilde{F}_{T}$ iff $(X, \mathbf{q})$ is $T$-transitive.

Proof: Assume $\tilde{F}_{T}$ holds, let $J=X$, and $\psi=\operatorname{id} X$. Let $x, y, z \in \dot{X}$, such that $\dot{x} \stackrel{q u}{\rightarrow} y$ and $\dot{y} \stackrel{q_{v}}{\rightarrow} z$. Define $\sigma: X \rightarrow \mathrm{U}(X)$ as follows : $\sigma(w)=\dot{w}$ for all $w \neq y$, and $\sigma(y)=\dot{x}$. Thus, $\kappa \sigma(\dot{y})=\dot{x}^{q_{\left(T_{\mu}, v\right)}} z$.

For the converse, let $J, \sigma, \psi$, and $\mathcal{F}$ be as in the statement of $\tilde{F}_{T}, \sigma(w) \stackrel{q q}{\rightarrow} \psi(w)$ for all $w \in J$, and $\psi \mathcal{F} \stackrel{q_{\mu}}{\rightarrow} z$ for some $z \in X$. Recall that a filter base for $\kappa \sigma \mathcal{F}$ is given by

$$
\left\{\bigcup_{y \in F} U_{y}: F \in \mathcal{F} \text { and } U_{y} \in \sigma(y)\right\}
$$

Let $\epsilon<T(\mu, \nu)$, and choose $\gamma<\mu, \theta<\nu$ such that $\epsilon<T(\gamma, \theta)<T(\mu, \nu)$. Let $D \in \kappa \sigma \mathcal{F}$; thus, $\bigcup_{y \in F} U_{y} \subseteq D$ for some $F \in \mathcal{F}$. Since $\psi \mathcal{F} \stackrel{q_{\mu}}{\rightarrow} z$ by hypothesis, there exists $b \in F$ such that $\dot{\psi(b)} \stackrel{q_{\mathcal{\gamma}}}{\rightarrow} z$, by $(\Delta)$. Since $\sigma(b) \stackrel{q_{v}}{\rightarrow} \psi(b),(\Delta)$ further implies that there exists $x \in U_{b} \subseteq D$ such that $\dot{x} \stackrel{q_{0}}{\rightarrow} \psi(b)$. Hence, $\dot{x} \stackrel{q_{T(\gamma, 0)}}{\rightarrow} z$ by $T$-transitivity, which implies $\dot{x} \stackrel{q_{e}}{\rightarrow} z$. Since $D$ and $\epsilon$ were chosen arbitrarily, $(\Delta)$ implies $\kappa \sigma \mathcal{F}^{q_{T(\mu, \nu)}} \underset{\longrightarrow}{\longrightarrow}$. 
Proposition 5.4 Let $(X, q)$ be a probabilistic convergence space which satisfies $(\Delta)$. Then, for any left-continuous $t$-norm $T,(X, \mathbf{q})$ satisfies $\tilde{R}_{T}$ iff $(X, \mathbf{q})$ is skew $T$-transitive.

Proof: Assume $\tilde{R}_{T}$ holds, let $J=X$, and $\psi=\mathrm{id}_{X}$. Let $x, y, z \in X$, such that $\dot{y} \stackrel{q_{\mu}}{\rightarrow} x$ and $\dot{y} \stackrel{q_{\nu}}{\rightarrow} z$. Define $\sigma: X \rightarrow \mathrm{U}(X)$ as follows : $\sigma(w)=\dot{w}$ for all $w \neq x$ and $\sigma(x)=\dot{y}$; hence, $\kappa \sigma(\dot{x})=\dot{y} \stackrel{q_{v}}{\rightarrow} z$. Therefore, $\psi \dot{x}=\dot{x} \stackrel{q^{T(\mu, \nu)}}{\longrightarrow} z$.

For the converse, let $J, \psi, \sigma$, and $\mathcal{F}$ be as in $\tilde{R}_{T}, \sigma(w) \stackrel{q_{\nu}}{\rightarrow} \psi(w)$ for all $w \in J$, and $\kappa \sigma \mathcal{F} \stackrel{q_{\mu}}{\longrightarrow} z$. Let $\epsilon<T(\mu, \nu)$, and choose $\gamma<\mu, \theta<\nu$ such that $\epsilon<T(\gamma, \theta)<T(\mu, \nu)$. Let $F \in \mathcal{F}$ and let $A_{F}=\left\{x \in X: \dot{x} \stackrel{q_{G}}{\rightarrow} \psi(j)\right.$, for some $\left.j \in F\right\}$. If $X \backslash A_{F} \in \kappa \sigma \mathcal{F}$, then there exists $G \in \mathcal{F}$ such that $\bigcup_{w \in G} U_{w} \subseteq X \backslash A_{F}$, where $U_{w} \in \sigma(w)$. Let $b \in F \cap G$. Since $\sigma(b) \stackrel{q_{w}}{\rightarrow} \psi(b)$, there exists $x \in U_{b} \subseteq X \backslash A_{F}$ such that $\dot{x} \stackrel{q_{9}}{\rightarrow} \psi(b)$, by axiom $(\Delta)$. But this implies $x \in A_{F}$, which is a contradiction; therefore, $A_{F} \in \kappa \sigma \mathcal{F}$. Since $A_{F} \in \kappa \sigma \mathcal{F}$, there exists a $y \in A_{F}$ such that $\dot{y} \stackrel{q_{q}}{\rightarrow} z$, by $(\Delta) ;$ but, also, $\dot{y} \stackrel{q_{o}}{\rightarrow} \psi(j)$ for some $j \in F$, by definition of $A_{F} \cdot \psi(j) \stackrel{q_{\text {T(o, }, 7)}}{\rightarrow} z$ follows by skew $T$-transitivity, and thus, $\psi(j) \stackrel{q_{e}}{\rightarrow} z$. Since $F$ and $\epsilon$ were chosen arbitrarily, $\psi \mathcal{F}^{q_{T(\mu, \nu)}} z$, by $(\Delta)$.

Corollary 5.5 Let $(X, q)$ be a probabilistic convergence space which satisfies $(\Delta)$. Then, for any left'-continuous $t$-norm $T,(X, \mathbf{q})$ satisfies $\tilde{R}_{T}$ iff $(X, \mathbf{q})$ is symmetric and $T$-transitive.

Proof: Use Proposition 5.2 and Proposition 5.4

\section{Generalized Metric Spaces as Probabilistic Convergence Spaces}

Definition 6.1 Let $\mathrm{X}$ be a set and let $d: X \times X \rightarrow[0, \infty]$, and consider the following "metric" axioms:

(d1) $d(x, x)=0$, for all $x \in X$;

(d2) $d(x, y) \leq d(x, z)+d(z, y)$ for all $x, y, z$ in $X$;

(d3) $d(x, y)=d(y, x)$, for all $x, y$ in $X$;

(d4) $d(x, y)=0$ implies $x=y$.

(d2) is known as the triangle inequality, $(d 3)$ as symmetry, and $(d 4)$ as separation. If $d$ is required to satisfy $(d 1),(d 2)$, and (d3) only (respectively $(d 1)$ and $(d 2)$ only, $(d 1)$ only), then $d$ is known as an extended pseudo-metric (respectively, extended pseudo-quasi-metric, extended pseudo-quasisemi-metric). A pair $(X, d)$, where $d$ is an extended pseudo-quasi-semi-metric on $\mathrm{X}$ is called an extended pseudo-quasi-semi-metric space or, more briefly, extended pqs-metric space. A mapping $f:(X, d) \rightarrow\left(Y, d^{\prime}\right)$ between extended pqs-metric spaces is called a contraction map if for all $x, y \in X$,

$$
d^{\prime}(f(x), f(y)) \leq d(x, y) .
$$

The category consisting of extended pseudo-quasi-semi-metric spaces as objects and contraction maps as morphisms will be denoted by $p q s-M E T^{\infty}$. The other cases are defined analagously.

We now identify those probabilistic convergence spaces which correspond to generalized metric spaces in $p q s-M E T^{\infty}, p q-M E T^{\infty}, p-M E T^{\infty}$ and $M E T^{\infty}$. Let $P P R S$ be the category of all 
probabilistic pretopological spaces. We denote by $P P R S(\Delta)$, the full subcategory of $P P R S$ whose objects satisfy the $(\Delta)$ axiom of Section 5 . We introduce three additional full subcategories of $P C S$ : $F_{T} P P R S(\Delta)$ : The $T$-topological objects in $P P R S(\Delta)$;

$R_{T} P P R S(\Delta):$ The $T$-regular objects in $P P R S(\Delta)$;

$R_{T} P P R S(\Delta)^{*}:$ The $\mathrm{T}_{2}$ objects in $R_{T} P P R S(\Delta)$.

Proposition 6.2 Let $T$ be a left-continuous $t$-norm, then the following hold:

(a) $R_{T} P P R S(\Delta) \subseteq F_{T} P P R S(\Delta)$.

(b) $\tilde{F}_{T} P C S \cap P P R S(\Delta)=F_{T} P P R S(\Delta)$.

(c) $\tilde{R}_{T} P C S \cap P P R S(\Delta)=R_{T} P P R S(\Delta)$.

Proof: To prove Part (a), apply Proposition 4.4, Proposition 5.3 and Corollary 5.5. For both Part (b) and Part (c), Proposition 4.4 will suffice.

Let $I$ denote the unit interval $[0,1]$, and let $S: I \rightarrow[0, \infty]$ be an order reversing homeomorphism; i.e., $S$ is a homeomorphism, and $S(\mu)>S(\nu)$ whenever $\mu, \nu \in I$ and $\mu<\nu$. The set of all such order reversing homeomorphisms $S$ is denoted by $\mathbf{S}$.

We now construct an isomorphism, based on a given $S \in \mathrm{S}$, between pqs-MET ${ }^{\infty}$ and $P P R S(\Delta)$. Let $S$ be in $S$. If $(X, d)$ is in pqs-MET $T^{\infty}$, let $\phi_{S}(d): F(X) \times I \rightarrow 2^{X}$ be defined as follows: For each $\mathcal{F} \in \mathbf{F}(X)$ and $\mu \in I, \phi_{S}(d)(\mathcal{F}, \mu)=\phi_{S}(d)_{\mu}(\mathcal{F})$, where $\phi_{S}(d)_{\mu}: \mathbf{F}(X) \rightarrow 2^{X}$ is defined by

$$
x \in \phi_{S}(d)_{\mu}(\mathcal{F}) \text { iff } \inf _{F \in \mathcal{F}} \sup _{y \in F} d(x, y) \leq S(\mu) .
$$

Alternatively, $\phi_{S}(d)_{\mu}$ can be described in an equivalent fashion by

$$
\mathcal{F} \stackrel{\phi s(d) \mu}{\rightarrow} x \text { iff } \inf _{F \in \mathcal{F}} \sup _{y \in F} d(x, y) \leq S(\mu)
$$

Proposition 6.3 If $(X, d)$ is in $\left|p q s-M E T^{\infty}\right|$, then $\left(X, \phi_{S}(d)\right)$ is in $|P P R S(\Delta)|$.

Proof: We first verify that, for all $\mu \in I, \phi_{S}(d)_{\mu}$ is a pretopology. (i) If $\mathcal{F}=\dot{x}$, then $\inf _{F \in \mathcal{F}} \sup _{y \in F} d(x, y)=d(x, x)=0$. Hence, $\dot{x} \stackrel{\phi_{s}(d)_{\mu}}{\rightarrow} x$ for all $\mu \in I$. (ii) If $\mathcal{F} \stackrel{\phi_{s}(d)_{\mu}}{\rightarrow} x$, and $\mathcal{G} \geq \mathcal{F}$, then $\inf _{G \in \mathcal{G}} \sup _{y \in G} d(x, y) \leq \inf _{F \in \mathcal{F}} \sup _{y \in F} d(x, y) \leq S(\mu)$. Thus, $\mathcal{G} \stackrel{\phi(d) \mu}{\rightarrow} x$. (iii) Given $x \in X$, let $\left\{\mathcal{F}_{j}: j \in J\right\}$ be the set of all filters on $X$ such that $\mathcal{F}_{j} \stackrel{\left.\phi_{s}(d)\right)_{\mu}}{\rightarrow} x$. Then, $\inf _{F \in \mathcal{F}}, \sup _{y \in F} d(x, y) \leq S(\mu)$ for all $j \in J$. Let an arbitrary element of $\bigcap_{j \in J} \mathcal{F}_{j}$ be denoted $\bigcup_{j \in J} F_{j}$, where each $F_{j}$ is in $\mathcal{F}_{j}$. It follows that

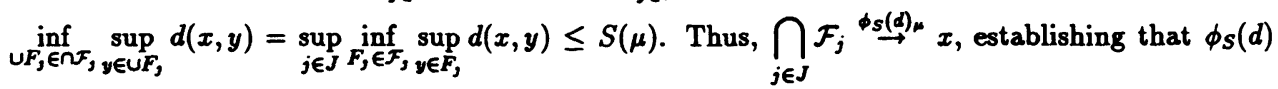
is pretopological. (PCS2): If $\mu=0$, then $\inf _{F \in \mathcal{F}} \sup _{y \in F} d(x, y) \leq S(\mu)=\infty$ for all $\mathcal{F} \in \mathbf{F}(X)$ and all $x \in X$; hence, $\mathcal{F} \stackrel{q_{0}}{\rightarrow} x$, for all $\mathcal{F} \in F(X)$ and all $x \in X$, and so $q_{0}$ is the indiscrete topology. (PCS3): Let $\mu \leq \nu$, and $\mathcal{F} \stackrel{\phi_{s}(d)_{\nu}}{\rightarrow} x$; then $\inf _{F \in \mathcal{F}} \sup _{y \in F} d(x, y) \leq S(\nu) \leq S(\mu)$. Hence, $\mathcal{F} \stackrel{\phi_{s}(d)_{\mu}}{\rightarrow} x$. (PCS4): To 
show $\phi_{S}(d)$ is left continuous, let $\mathcal{F}^{\phi_{S}(d)_{\mu}} \rightarrow$ for all $\mu<\nu$. Then, $\inf _{F \in \mathcal{F}} \sup _{y \in F} d(x, y) \leq S(\mu)$ for all $\mu<\nu$ implies $\inf _{F \in \mathcal{F}} \sup _{y \in F} d(x, y) \leq S(\nu)$, and thus, $\mathcal{F}^{\phi_{S(d)}} \stackrel{\text {. }}{\rightarrow} x$.

It remains to show that $\phi_{S}(d)$ satisfies $(\Delta)$. Let $U$ be an ultrafilter on $X$ and let $\nu \in I$. Assume. $\mathcal{U} \stackrel{\phi_{S}(d) \nu}{\rightarrow} x$; then $\inf _{U \in \mathcal{U}} \sup _{y \in U} d(x, y) \leq S(\nu)$. Hence, if $\mu<\nu$, there exists a $V \in \mathcal{U}$ such that $\sup _{y \in V} d(x, y) \leq S(\mu)$, which implies $d(x, y) \leq S(\mu)$ for all $y \in V$. It follows that if $U$ is any element of $U$, there exists a $y \in U \cap V$, and hence a $y \in U$, such that $\dot{y} \stackrel{\phi s(d) \mu}{\rightarrow} x$.

Conversely, let $\mathcal{U}$ be an ultrafilter on $X$ and let $\nu \in I$. Assume that for all $U \in \mathcal{U}$ and all $\mu<\nu$, there exists a $y \in U$ such that $\dot{y} \stackrel{\phi(d))_{\mu}}{\rightarrow} x$. For each $\mu<\nu$, put $A_{\mu}=\left\{y \in X: \dot{y} \stackrel{\phi s(d)_{\mu}}{\rightarrow} x\right\}$; then $X-A_{\mu} \notin \mathcal{U}$, and so $A_{\mu} \in \mathcal{U}$ for each $\mu<\nu$. It follows that $\inf _{U \in \mathcal{U}} \sup _{y \in U} d(x, y) \leq \inf _{\mu<\nu} \sup _{y \in A_{\mu}} d(x, y) \leq S(\nu)$. Therefore, $\mathcal{U} \stackrel{\phi_{S}(d) \nu}{\rightarrow} x$, and consequently, $\phi_{S}(d)$ satisfies $(\Delta)$.

Let $\phi_{S}$ be defined for objects by $\phi_{S}(X, d)=\left(X, \phi_{S}(d)\right)$ and for morphisms by $\phi_{S}(f)=f$.

Proposition $6.4 \phi_{S}: p q s-M E T^{\infty} \rightarrow P P R S(\Delta)$ is a functor.

Proof: Let $(X, d)$ and $\left(X^{\prime}, d^{\prime}\right)$ be objects in pqs-MET $T^{\infty}, f:(X, d) \rightarrow\left(X^{\prime}, d^{\prime}\right)$ a contraction map and $\mathcal{F} \in \mathbf{F}(X)$. If $\mu \in I$, and $\mathcal{F} \stackrel{\phi^{s}(d) \mu}{\rightarrow} x$, then

$$
\inf _{H \in f(\mathcal{F})} \sup _{w \in H} d^{\prime}(f(x), w) \leq \inf _{F \in \mathcal{F}} \sup _{y \in F} d^{\prime}(f(x), f(y)) \leq \inf _{F \in \mathcal{F}} \sup _{y \in F} d(x, y) \leq S(\mu) .
$$

Hence, $f(\mathcal{F}) \stackrel{\phi_{S}\left(d^{\prime}\right) \mu}{\rightarrow} f(x)$, establishing that $f:\left(X, \phi_{S}(d)\right) \rightarrow\left(X^{\prime}, \phi_{S}\left(d^{\prime}\right)\right)$ is continuous.

We next construct the inverse functor $\psi_{S}: P P R S(\Delta) \rightarrow p q s-M E T^{\infty}$. If $(X, q)$ is in $|P P R S(\Delta)|$, define $\psi_{S}(X, \mathbf{q})=\left(X, \psi_{S}(\mathbf{q})\right)$, where $\psi_{S}(\mathbf{q}): X \times X \rightarrow[0, \infty]$ is given by

$$
\psi_{S}(\mathbf{q})(x, y)=\inf \{S(\nu): \dot{y} \stackrel{q}{\rightarrow} x\} .
$$

If $f$ is a morphism in $\operatorname{PPRS}(\Delta)$, define $\psi_{S}(f)=f$.

Proposition 6.5 If $(X, \mathbf{q}) \in|P P R S(\Delta)|$, then $\left(X, \psi_{S} q\right) \in\left|p q s-M E T^{\infty}\right|$.

Proof: It is sufficient to show that $\psi_{S}(\mathbf{q})$ satisfies $(d 1)$ of Definition 6.1. Since $\dot{x} \stackrel{q_{1}}{\rightarrow} x$, for all $x \in X, \psi_{S}(\mathbf{q})(x, x)=\inf \left\{S(\nu): \dot{x} \stackrel{q_{\nu}}{\longrightarrow} x\right\}=S(1)=0$, for all $x \in X$.

Proposition 6.6 $\psi_{S}: P P R S(\Delta) \rightarrow p q s-M E T^{\infty}$ is a functor.

Proof: Let $(X, \mathbf{q})$ and $\left(X^{\prime}, \mathbf{q}^{\prime}\right)$ be objects in $P P R S(\Delta), f:(X, \mathbf{q}) \rightarrow\left(X^{\prime}, \mathbf{q}^{\prime}\right)$ a continuous function, and $x, y \in X$. Select $\mu \in I$ such that $\psi_{S}(\mathbf{q})(x, y)=S(\mu)$; then $\dot{y} \stackrel{q_{\mu}}{\rightarrow} x$, which implies $\dot{f(y)} \stackrel{q_{\mu}^{\prime}}{\rightarrow} f(x)$, by continuity of $f$. Hence, $\psi_{S}\left(\mathbf{q}^{\prime}\right)(f(x), f(y)) \leq S(\mu)=\psi_{S}(\mathbf{q})(x, y)$, and thus, $f:\left(X, \psi_{S}(\mathbf{q})\right) \rightarrow\left(X^{\prime}, \psi_{S}\left(\mathbf{q}^{\prime}\right)\right)$ is a contraction map.

Theorem $6.7 \phi_{S}: p q s-M E T^{\infty} \rightarrow P P R S(\Delta)$ is an isomorphism. 
Proof: It will be sufficient to show $\psi_{S} \circ \phi_{S}(X, d)=(X, d)$ for each $(X, d) \in p q s-M E T^{\infty}$, and $\phi_{S} \circ \psi_{S}(X, \mathbf{q})=(X, \mathbf{q})$ for each $(X, \mathbf{q}) \in P P R S(\Delta)$. Let $(X, d)$ be in pqs-MET $T^{\infty}$ and $x, y \in$ $X . \psi_{S}\left(\phi_{S}(d)\right)(x, y)=\inf \left\{S(\nu): \dot{y} \stackrel{\phi_{s}(d) \nu}{\rightarrow} x\right\}=\inf \{S(\nu): d(x, y) \leq S(\nu)\}=d(x, y)$. Hence, $\psi_{S} \circ \phi_{S}(X, d)=(X, d)$.

Now let $(X, \mathbf{q})$ be in $\operatorname{PPRS}(\Delta), \nu \in I$ and $\mathcal{U}$ an ultrafilter on $X$. Let $\mathcal{U} \stackrel{q u}{\rightarrow} x$; then by axiom $(\Delta)$, for all $\mu<\nu$ and all $U \in \mathcal{U}$, there exists a $y \in U$ such that $\dot{y} \stackrel{q_{\mu}}{\rightarrow} x$. For each $\mu<\nu$, put $A_{\mu}=\left\{y \in X: \dot{y} \stackrel{q_{\mu}}{\rightarrow} x\right\}$. Then $X-A_{\mu} \notin \mathcal{U}$, and so $A_{\mu} \in \mathcal{U}$, for all $\mu<\nu$. It follows that $\inf _{U \in \mathcal{U}} \sup _{y \in U} \psi_{S}(\mathbf{q})(x, y) \leq \inf _{\mu<\nu} \sup _{y \in A_{\mu}} \psi_{S}(\mathbf{q})(x, y) \leq S(\nu)$. Therefore, $U^{\phi_{S}\left(\psi_{S}(\mathbf{q})\right)_{\nu}} x$, and thus $\phi_{\boldsymbol{S}}\left(\psi_{\boldsymbol{S}}(\mathbf{q})\right) \leq \mathbf{q}$.

To establish the reverse inequality, let $\left(X, \phi_{S}\left(\psi_{S}(\mathbf{q})\right)\right)$ be in $P P R S(\Delta), \nu \in I$ and $\mathcal{U}$ an ultrafilter on $X$. Let $\mathcal{U}^{\phi_{S}\left(\psi_{S}(\mathbf{q})\right)_{\nu}} x$; then $\inf _{U \in \mathcal{U}} \sup _{\mathbf{y} \in U} \psi_{S}(\mathbf{q})(x, y) \leq S(\nu)$. Hence, if $\mu<\nu$, there exists a $V \in \mathcal{U}$ such that $\sup _{y \in V} \psi_{S}(\mathbf{q})(x, y) \leq S(\mu)$, which implies $\psi_{S}(\mathbf{q})(x, y) \leq S(\mu)$ for all $y \in V$. It follows that if $U$ is any element of $U$, there exists a $y \in U \cap V$ such that $\dot{y} \stackrel{q \mu}{\rightarrow} x$. By axiom $(\Delta)$, it follows that $\mathcal{U} \stackrel{q_{v}}{\rightarrow} x$, and consequently, $\mathbf{q} \leq \phi_{S}\left(\psi_{S}(\mathbf{q})\right)$. Hence, $\mathbf{q}=\phi_{S} \circ \psi_{S}(\mathbf{q})$.

A useful class of continuous $t$-norms may be obtained via the next proposition. See Chapter 5 of [16] for additional information.

Proposition 6.8 Let $S$ be in $S$. Then the mapping $T_{S}: I^{2} \rightarrow I$, defined by $T_{S}(\mu, \nu)=S^{-1}(S(\mu)+$ $S(\nu))$ is a continuous $t$-norm.

Any continuous $t$-norm $T$ of the form $T=T_{S}$ for some $S \in \mathbf{S}$ is called the strict $t$-norm derived from $S$.

In the results that follow, let $S$ be in $\mathbf{S}$, and let $T$ be the strict $t$-norm derived from $S$. Let $\tilde{\phi}_{S}$ be the restriction of $\phi_{S}$ to $p q-M E T^{\infty}$. We now show that $\tilde{\phi}_{S}: p q-M E T^{\infty} \rightarrow F_{T} P P R S(\Delta)$ is an isomorphism.

Proposition 6.9 If $(X, d) \in\left|p q-M E T^{\infty}\right|$, then $\left(X, \phi_{S}(d)\right)$ is $T$-transitive.

Proof: Let $x, y, z \in X, \dot{x} \stackrel{\left.\phi^{(d)}\right)}{\rightarrow} y$, and $\dot{y} \stackrel{\phi s(d)_{\nu}}{\rightarrow} z$. Then, $d(x, y) \leq S(\mu)$, and $d(y, z) \leq S(\nu)$. By the triangle inequality, $d(x, z) \leq S(\mu)+S(\nu)=S(T(\mu, \nu))$; hence, $\dot{z} \stackrel{\phi S(d)_{T(\mu, \nu)}}{\longrightarrow}$.

Corollary 6.10 If $(X, d) \in\left|p q-M E T^{\infty}\right|$, then $\left(X, \phi_{S}(d)\right) \in\left|F_{T} P P R S(\Delta)\right|$

Proof: $\left(X, \phi_{S}(d)\right) \in|P P R S(\Delta)|$, by Proposition 6.3 , and $\left(X, \phi_{S}(d)\right)$ satisfies $\tilde{F}_{T}$ by Proposition 5.3. Hence, $\left(X, \phi_{S}(d)\right) \in\left|F_{T} P P R S(\Delta)\right|$, by Proposition 6.2(b).

Proposition 6.11 If $(X, \mathbf{q}) \in\left|F_{T} P P R S(\Delta)\right|$, then $\left(X, \psi_{S}(\mathbf{q})\right) \in\left|p q-M E T^{\infty}\right|$.

Proof: It is sufficient to show $\left(X, \psi_{S}(q)\right)$ satisfies (d2) of Definition 6.1 (the triangle inequality). Let $x, y, z \in X$. Select $\mu, \nu \in I$ such that $d(x, y)=S(\mu)$, and $d(y, z)=S(\nu)$; thus, $\dot{y} \stackrel{9 \mu}{\rightarrow} x$ and $\dot{z} \stackrel{q_{\nu}}{\rightarrow} y$. It follows that $\dot{z}^{q_{T(\mu, \nu)}} \underset{\sim}{\longrightarrow}$, by Proposition 5.3, and therefore,

$$
d(x, z) \leq S(T(\mu, \nu))=S(\mu)+S(\nu)=d(x, y)+d(y, z) .
$$


Combining the three previous results we obtain the next theorem.

Theorem 6.12 $\tilde{\phi}_{S}: p q-M E T^{\infty} \rightarrow F_{T} P P R S(\Delta)$ is an isomorphism.

Now let $\hat{\phi}_{S}$ be the restriction of $\phi_{S}$ to $p-M E T^{\infty}$. Following a similar procedure, we show that the mapping $\hat{\phi}_{S}: p-M E T^{\infty} \rightarrow R_{T} P P R S(\Delta)$ is an isomorphism.

Proposition 6.13 If $(X, d) \in\left|p-M E T^{\infty}\right|$, then $\left(X, \phi_{S}(d)\right)$ is symmetric.

Proof: Let $x, y \in X, \mu \in I$, such that $\dot{x} \stackrel{\left.\phi_{s}(d)\right)_{\mu}}{\rightarrow} y$. Then, using (d3) of Definition 6.1, $d(x, y)=$ $d(y, x) \leq S(\mu)$, which implies $\dot{y} \stackrel{\phi s(d) \mu}{\rightarrow} x$.

Corollary 6.14 If $(X, d) \in\left|p-M E T^{\infty}\right|$, then $\left(X, \phi_{S}(d)\right) \in\left|R_{T} P P R S(\Delta)\right|$.

Proof: $\left(X, \phi_{S}(d)\right) \in|P P R S(\Delta)|$, by Proposition 6.3. Furthermore, $\left(X, \phi_{S}(d)\right)$ is $T$-transitive by Proposition 6.9 , and thus, $\left(X, \phi_{S}(d)\right)$ satisfies $\tilde{R}_{T}$ by Proposition 6.13 and Corollary 5.5. Hence, $\left(X, \phi_{S}(d)\right) \in\left|R_{T} P P R S(\Delta)\right|$ by Proposition 6.2(c).

Proposition 6.15 If $(X, \mathbf{q}) \in\left|R_{T} P P R S(\Delta)\right|$, then $\left(X, \psi_{S}(\mathbf{q})\right) \in\left|p-M E T^{\infty}\right|$.

Proof: We must prove that $\left(X, \psi_{S} q\right)$ satisfies $(d 3)$ of Definition 6.1. $(X, q)$ is symmetric by Corollary 5.5. It follows that, for all $x, y \in X, \psi_{S}(\mathbf{q})(x, y)=S(\mu) \Rightarrow \dot{y} \stackrel{q_{\mu}}{\rightarrow} x \Rightarrow \dot{x} \stackrel{q_{\mu}}{\rightarrow} y \Rightarrow$ $\psi_{S}(\mathbf{q})(y, x) \leq S(\mu)$. Hence, $\psi_{S}(\mathbf{q})(y, x) \leq \psi_{S}(\mathbf{q})(x, y)$, and by a similar argument, $\psi_{S}(\mathbf{q})(y, x) \leq$ $\psi_{S}(\mathbf{q})(x, y)$. Thus, $\psi_{S}(\mathbf{q})(y, x)=\psi_{S}(\mathbf{q})(x, y)$.

Theorem 6.16 $\hat{\phi_{S}}: p-M E T^{\infty} \rightarrow R_{T} P P R S(\Delta)$ is an isomorphism.

Recall that $R_{T} P P R S(\Delta)^{*}$ is the full subcategory of $R_{T} P C S(\Delta)$ consisting of those objects which satisfy the $T_{2}$ property. Let $\dot{\phi}_{S}$ be the restriction of $\phi_{S}$ to $M E T^{\infty}$. The next results show that $\check{\phi_{S}}: M E T^{\infty} \rightarrow R_{T} P P R S(\Delta)^{*}$ is an isomorphism.

Proposition 6.17 If $(X, d) \in\left|M E T^{\infty}\right|$, then $\left(X, \phi_{S}(d)\right)$ is $\mathbf{T}_{2}$.

Proof: Let $x, y \in X, \dot{x} \stackrel{\phi_{s}(d)}{\rightarrow} y$. Then, $d(x, y)=S(1)=0$, which implies $x=y$, by $(d 4)$ of Definition 6.1. Thus, $\left(X, \phi_{S}(d)\right)$ is $\mathbf{T}_{1}$, and so $\left(X, \phi_{S}(d)\right)$ is $\mathbf{T}_{2}$ by Proposition 4.5.

Corollary 6.18 If $(X, d) \in\left|M E T^{\infty}\right|$, then $\left(X, \phi_{S}(d)\right) \in\left|R_{T} P P R S(\Delta)^{*}\right|$

Proposition 6.19 If $(X, \mathrm{q}) \in\left|R_{T} P P R S(\Delta)^{*}\right|$, then $\left(X, \psi_{s}(\mathbf{q})\right) \in\left|M E T^{\infty}\right|$.

Proof: Let $x, y \in X, \psi_{S} q(x, y)=0$. Then, $\dot{y} \stackrel{q_{1}}{\rightarrow} x$, but, since $(X, \mathbf{q})$ is $\mathbf{T}_{1}$, we must have $x=y$ since $\left(X, q_{1}\right)$ is $\mathbf{T}_{1}$. Therefore, $\left(X, \psi_{S} \mathbf{q}\right)$ satisfies $(d 4)$ of Definition 6.1. 
Theorem 6.20 If $S \in \mathrm{S}$ and $T$ the $t$-norm derived from $S$, then

$$
\check{\phi_{S}}: M E T^{\infty} \rightarrow R_{T} P P R S(\Delta)^{*}
$$

is an isomorphism.

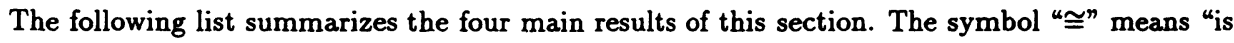
isomorphic to."

(1) $p q s-M E T^{\infty} \cong P P R S(\Delta)$;

(2) $p q-M E T^{\infty} \cong F_{T} P P R S(\Delta)$;

(3) $p-M E T^{\infty} \cong R_{T} P P R S(\Delta)$;

(4) $M E T^{\infty} \cong R_{T} P P R S(\Delta)^{*}$.

\section{References}

[1] H.J. Biesterfeldt, Jr., "Regular Convergence Spaces," Indag. Math. 28 (1966), 605-607.

[2] P. Brock and D. Kent, "Probabilistic Convergence Spaces and Regularity," Intl. J. Math. and Math. Sci., to appear.

[3] P. Brock and D. Kent, "Approach Spaces, Limit Tower Spaces and Probabilistic Convergence Spaces," Applied Categorical Structures, to appear.

[4] P. Brock, Probabilistic Convergence Spaces," Doctoral Dissertation, Washington State University, 1996.

[5] C. H. Cook and H. R. Fischer, "Regular Convergence Spaces", Math Ann. 174 (1967), 1-7.

[6] L.C. Florescu, "Probabilistic Convergence Structures," Aequationes Math. 38 (1989), 123-145.

[7] M. J. Frank, "Probabilistic Topological Spaces," J. Math. Analysis and Appl. 34 (1971), 67-81.

[8] D. Kent and G. Richardson, "Convergence Spaces and Diagonal Conditions, "Topology and Its Applications, to appear.

[9] H. J. Kowalsky, "Limesräume und Komplettierung," Math. Nachr. 12 (1954), 301-340.

[10] E. Lowen and R. Lowen, "A Quasitopos Containing CONV and MET as Full Subcategories," Intl. J. Math. and Math. Sci. 11 (1988) 417-438.

[11] R. Lowen, "Approach Spaces: A Common Supercategory of TOP and MET," Math. Nachr. 141 (1989), 183-226.

[12] K. Menger, "Statistical Metrics," Proc. Natl. Acad. Sci. USA, 37 (1951), 178-180. 
[13] G. Preuss, "Theory of Topological Structures," Kluwer Academic Publishers, 1987.

[14] G. D. Richardson and D. C. Kent, "Probabilistic Convergence Spaces" J. Austral. Math. Soc., to appear.

[15] B. Schweizer and A. Sklar, "Statistical Metric Spaces," Pacific J. Math. 10 (1960) 313-334.

[16] B. Schweizer and A. Sklar, Probabilistic Metric Spaces, North Holland Publ. Co. New York 1983.

[17] O. Wyler, "Convergence Axioms for Topology," to appear. 


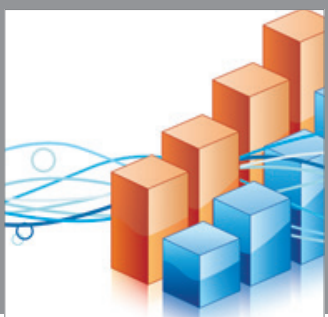

Advances in

Operations Research

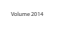

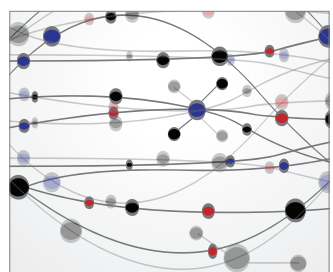

\section{The Scientific} World Journal
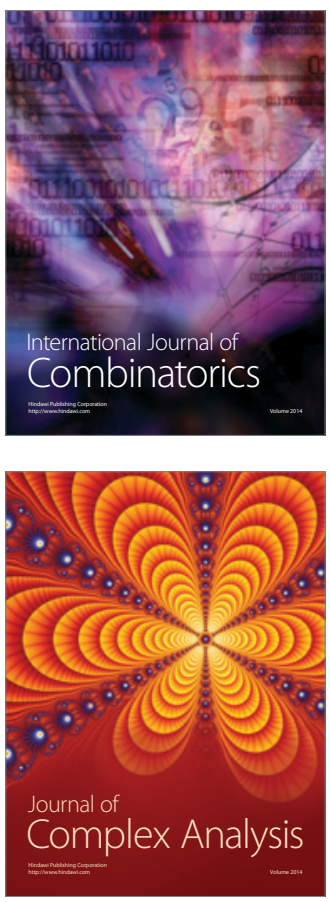

International Journal of

Mathematics and

Mathematical

Sciences
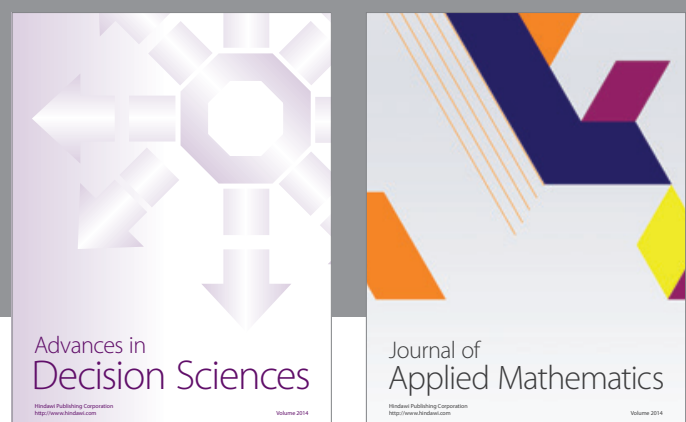

Journal of

Applied Mathematics
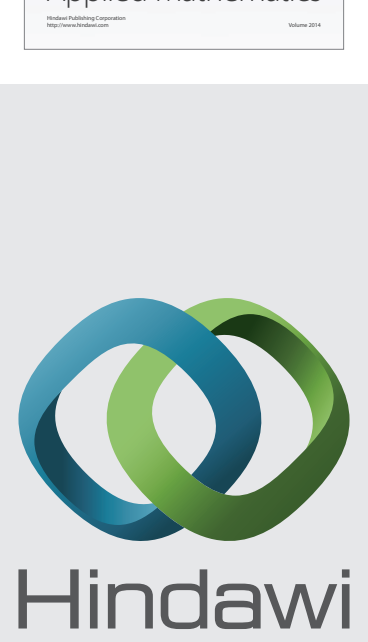

Submit your manuscripts at http://www.hindawi.com
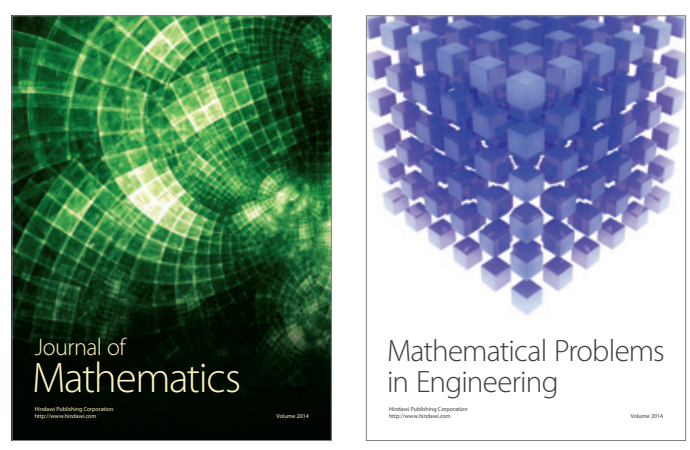

Mathematical Problems in Engineering
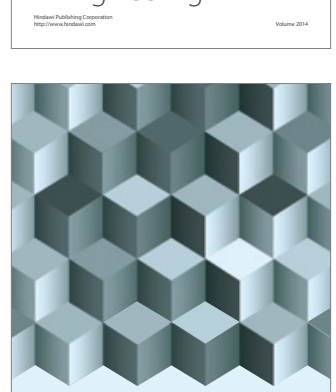

Journal of

Function Spaces
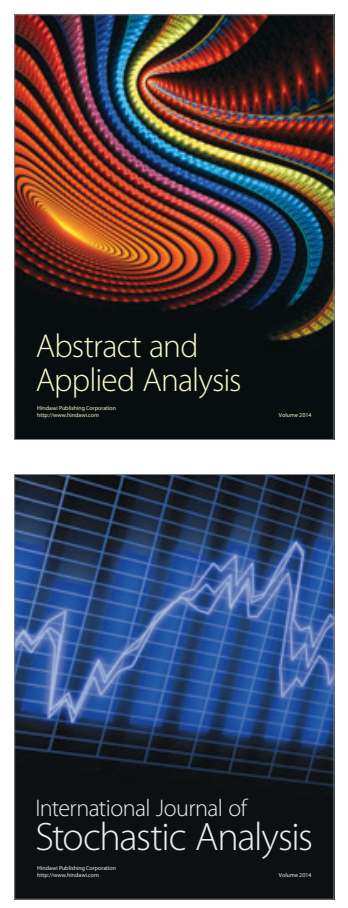

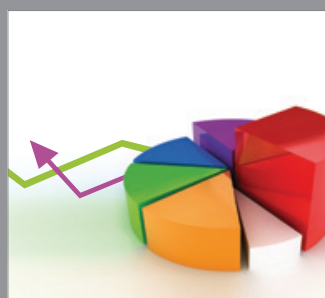

ournal of

Probability and Statistics

Promensencen
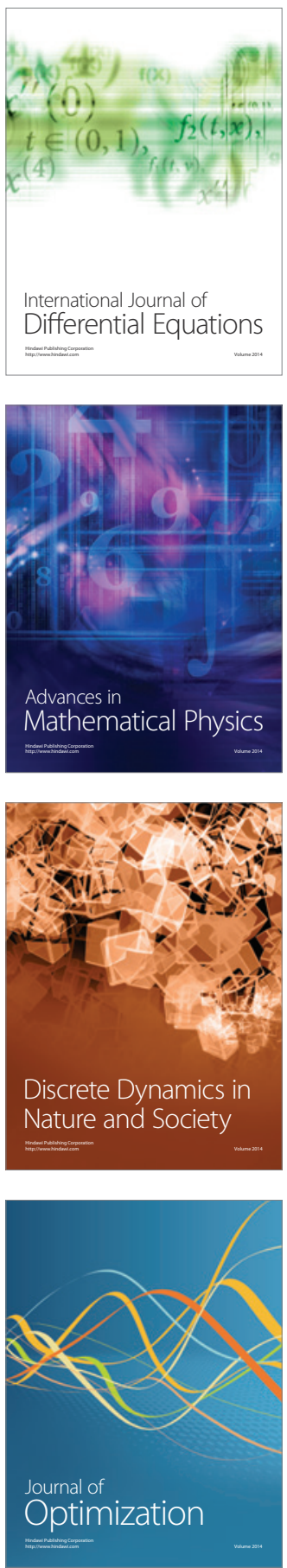